\title{
Etching and Polymerization Reactions of Alkoxythiophenes in HKUST-1: Choosing Between Filled and Core-Shell MOF/Polymer Composite Structures
}

William James, Colette M. Sullivan, and Nicholas Marshall ${ }^{*}$

University of South Carolina Aiken, Department of Chemistry and Physics, 471 University Parkway, Aiken, SC 29801

*To whom correspondence should be addressed. nicholasm@usca.edu. ORCID 0000-0001-8048-0857

\section{Abstract}

In an earlier report, our group found that neat 3,4-ethylenedioxythiophene (EDOT) directly reacts with the Cu-based MOF HKUST-1 to form a core-shell composite in which the HKUST-1 surface is coated with conjugated polymer, while unsubstituted thiophenes formed a filled composite where polythiophene is contained in the MOF's pores. In this work, we found that EDOT and 3-methoxythiophene (3MOT), which react in the neat form to form core-shell composites, can be loaded into HKUST-1 pores without reaction when dissolved in hexanes. To a lesser extent, 3-hexyloxythiophene (3HOT) can be loaded in the same way. These hexane-loaded composites can then be heated, resulting in the formation of a 'filled' composite. This finding allows us to choose to prepare either a core-shell or a filled composite by selecting reaction conditions. The process of heating with alkoxythiophenes results in a morphology change in the HKUST-1 material, at a surprisingly low temperature. Composites were characterized by nitrogen sorption measurements, powder X-ray diffraction, and vibrational spectroscopy.

\section{Introduction}

MOFs are interesting due to potential applications in catalysis, environmental remediation, and energy. ${ }^{1,2}$ In particular, as a result of a U.S. Department of Energy initiative to promote hydrogen fuel applications, gas adsorption in MOFs has been thoroughly studied. ${ }^{3,4}$ However, studies of MOFs as catalysts and as ingredients in modern metamaterials are proliferating rapidly. Our interest in MOFs initially began as we sought to use them as a physical template for making conjugated polymer materials with nanoscale structure. We originally stumbled upon the direct reaction between HKUST-1 and thiophenes (Scheme 1) as part of that project, ${ }^{5}$ in which we combined monomers with HKUST-1 and observed a visible reaction marked by color change and heat evolution. This reaction turned out to be an oxidative polymerization, directly initiated by the MOF but with atmospheric oxygen as a possible terminal oxidant.

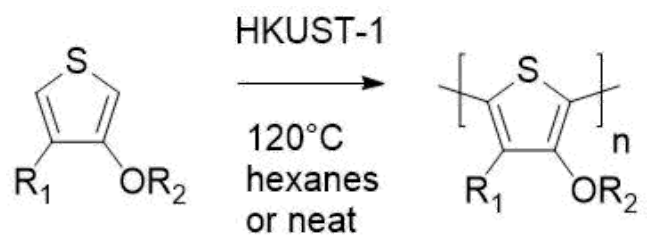

Scheme 1. Alkoxythiophenes react directly with the metal-organic framework HKUST-1 to form a composite with the resulting polythiophene. 
Several groups, including Uemura ${ }^{6-8}$ and coworkers, have made composites in which MOF pores contain conjugated polymers. The most common approach works by soaking monomers into a MOF and crosslinking the entrapped monomer units by application of an external oxidizer. As of the present date, a few reports have described direct reactions between a nanoporous structure and monomers. ${ }^{6,9,10}$ To the best of our knowledge, though, our 2019 report was the first in which a "core-shell" structure was reported, with the conjugated polymer PEDOT forming primarily on the surface of the MOF. Core-shell structures involving MOFs have many potential uses, including controlled-release applications and stabilization of the MOF lattice structure. ${ }^{11,12}$ Our goal in the present study was to improve our control over the MOF/monomer reaction, and develop the capacity to choose a "filled" or a "core-shell" structure by selecting the reaction conditions. In this report, we describe our principal finding that two monomers, 3,4-ethylenedioxythiophene (EDOT) and 3-methoxythiophene (3MOT), can be induced to form either a core-shell structure or a filled composite by direct reaction with the MOF HKUST-1 by changing reaction conditions. These electron-rich arenes form a core-shell coating on the surface of HKUST-1 crystals in the neat liquid form, but can be loaded into the pores of HKUST-1 without reaction as a solution in hexanes. Heating the monomer-loaded MOF in air yields a polythiophene-filled composite framework without visible signs of surface-bound polymer. We believe that this finding will enable the creation of new architectures based on the combination of conjugated polymers and redox-active MOFs.

\section{Materials and Methods}

HKUST-1 was prepared in our lab by the well-known solvothermal synthesis ${ }^{13}$ with trimesic acid and copper acetate in DMF/water, in a simple Teflon screw-pipe autoclave. Immediately before use, the MOF was activated by overnight heating in a vacuum oven. After activating, the MOF was a visible deep purple color.

Synthesis of alkoxythiophenes. EDOT and 3MOT were obtained from Alfa Aesar and used as received. 3HOT was prepared by the common method of transetherification from $3 \mathrm{MOT}^{14}$ followed by distillation under reduced pressure.

Preparation of hexane-loaded composites. A $0.5 \mathrm{M}$ solution of each monomer was prepared in hexanes. A $5 \mathrm{~mL}$ portion of this solution was added to a $100 \mathrm{mg}$ aliquot of activated MOF in a scintillation vial, and the vial was sealed. After incubating overnight at room temperature, the suspension was vortexed and the solid collected by vacuum filtration on a fritted funnel. The solid was washed with 3 portions of fresh hexane. For characterization of loaded pre-polymerization MOFs, the resulting material was used as formed. For composite formation, the isolated solid was immediately transferred to a laboratory oven and heated to $120^{\circ} \mathrm{C}$ for 1 hour. Between uses, all materials were stored in a scintillation vial under lab atmosphere without any other isolation from oxygen or water.

Vibrational spectroscopy. Infrared spectra were acquired on a Nicolet 380 spectrometer with a Smart Orbit diamond ATR attachment. 64 scans were summed at a $4 \mathrm{~cm}^{-1}$ resolution to produce a typical spectrum. Raman spectra were acquired on a Renishaw InVia Raman microscope using a 
$488 \mathrm{~nm}$ laser, typically at $1 \%$ maximum power and an $1 \mathrm{~s}$ exposure time. Selected spectra were repeated using the $785 \mathrm{~nm}$ laser to check for artifacts. Spectra shown are individual (single) scans.

Powder X-ray diffraction. PXRD measurements were taken at the University of South Carolina Department of Chemistry and Biochemistry's facility on a Rigaku Ultima IV Powder X-ray diffraction system (Cu Ka radiation) with a diffracted beam graphite monochromator.

Nitrogen adsorption studies. $\mathrm{N}_{2}$ adsorption isotherms were measured at the Applied Research Center facility, Aiken, SC, USA, using a Quantachrome Novatouch 2LX instrument after degassing at 200 degrees $C$ for $24 \mathrm{~h}$. Fitting of the volume / reduced pressure curve was performed using the manufacturer's TouchWin software and the DFT model.

Scanning electron microscopy. SEM images were acquired and EDX spectra/element mapping was performed on an Hitachi Cold Field Emission 8200 Series FE-SEM at the Applied Research Center facility, Aiken, SC, USA. A $2 \mathrm{kV}$ accelerating voltage was used for typical images shown.

\section{Results and Discussion}

During the preparation of composites, we immediately observed that alkoxythiophene-loaded MOFs turn a vivid black color upon heating. This color change is most dramatic in hexane-loaded composites, which retain the typical purple-blue color of HKUST-1 with a guest molecule until heated, and then turn black almost immediately upon heating. Black, of course, is a color attainable by highly doped polythiophenes of most kinds including unsubstituted polythiophene and EDOT. ${ }^{15}$ Because of this phenomenon, we generally find a darkening of the MOF composite after reaction with arene monomer to be a good initial screening for whether a composite actually forms. However, color does not reliably indicate the difference between a "core" and "shell" structure, as both appear black to the naked eye, although visible light absorption spectroscopy can distinguish between the two by observation of the redshift of the LMCT transition appearing at $480 \mathrm{~nm}$ in pristine HKUST-1. Encouragingly, we observe a similar shift in the case of hexane-loaded 3MOT composite, but not the neat 3MOT composite, in the present study. (Figure S9)

As an initial feasibility check in our attempts to adapt the EDOT/HKUST-1 reaction to prepare a filled composite, we compared Raman spectra of HKUST-1 samples loaded with hexanes/EDOT solution before and after heating. (Figure 1) We adopted this approach with the understanding that it was unlikely that polymer would be formed on the surface of the hexane-loaded material after heating if it were not already present before heating (i.e., if it did not form at room temperature) The results were encouraging. Raman spectroscopy of hexane-loaded EDOT shows a clean spectrum with narrow bands which is a good match for the reported spectrum of the free monomer ${ }^{16}$ plus HKUST-1 before polymerization, but after heating shows a fluorescence background and broadening and shifting of monomer peaks consistent with the formation of PEDOT, particularly including the C-O-C deformations of various oxidation states of PEDOT near $440 \mathrm{~cm}^{-1}$ and 578 $\mathrm{cm}^{-1}$. 


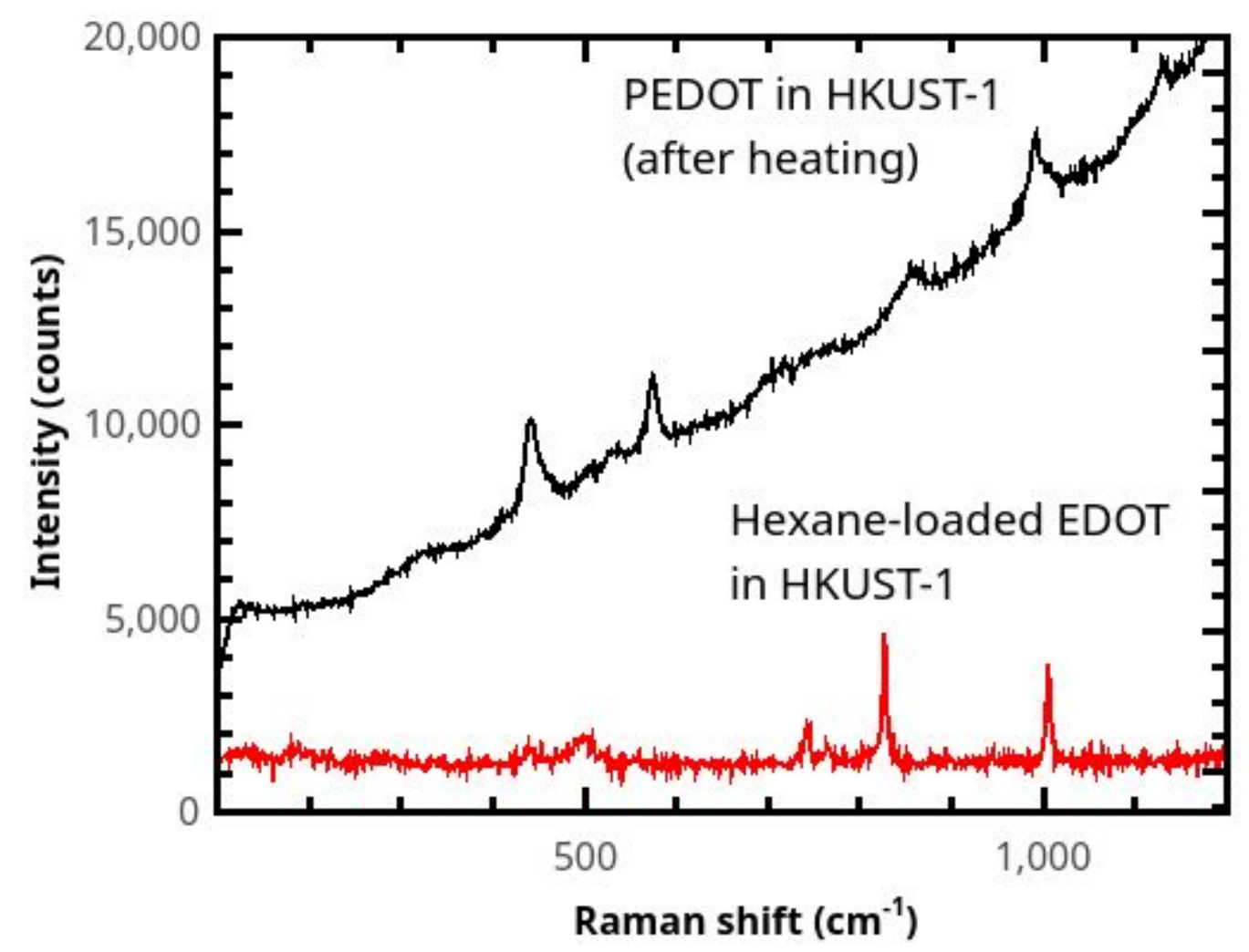

Figure 1. The Raman spectrum of EDOT loaded into the MOF HKUST-1 in hexane shows a peak corresponding to the out-of-plane $\mathrm{C}-\mathrm{H}$ bend $\left(830 \mathrm{~cm}^{-1}\right)$ of monomeric EDOT before heating, but fluorescence background and the appearance of new C-O-C deformation peaks near 440 and 580 $\mathrm{cm}^{-1}$ due to PEDOT after heating.

IR and Raman spectra of composites show some distinction between hexane-loaded composites and those prepared with neat monomer. Hexane-loaded PEDOT composites showed typical peaks for polythiophenes, especially the narrow $C=C$ asymmetric stretch of polythiophene near $1420 \mathrm{~cm}^{-1}$, but not the dramatic difference due to surface polymer (also observed in a prior study ${ }^{5}$ ) with EDOT formed at the HKUST-1 surface. (Figure 2) 3MOT shows the same distinction in IR, with a very broad absorption covering the entire measured spectrum below $1800 \mathrm{~cm}^{-1}$ due to superficial conjugated polymer. 

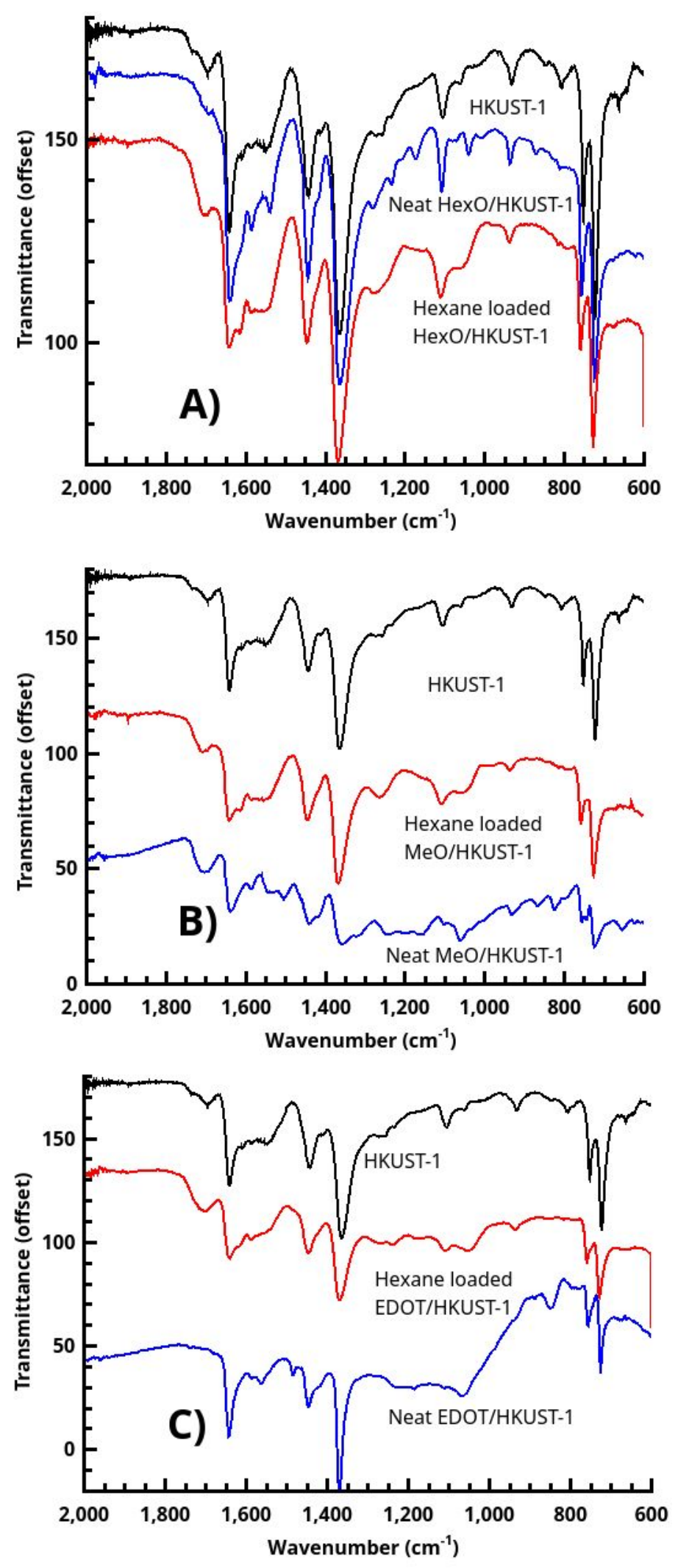

Figure 2. (A) HKUST-1 modified with neat 3-hexyloxythiophene shows little difference by IR vs. the pristine MOF, but when 3HOT is loaded in hexane, new bands appear near 1500 and $1100 \mathrm{~cm}^{-1}$ corresponding to P3HOT. (B) By contrast to $A$, the IR spectrum of HKUST with neat 3-methoxythiophene is dominated by polymer, but distinct bands corresponding to polymer modes 
are seen overlaid on the MOF spectrum in the hexane-loaded material. (C) Similarly to (B), the spectrum of neat EDOT/HKUST-1 is dominated by the polymer, but both polymer and MOF peaks can be seen in the hexane-loaded material.

\begin{tabular}{|c|c|c|}
\hline Material & $\begin{array}{c}\text { Sulfur } \\
\text { percentage }\end{array}$ & $\begin{array}{c}\text { Copper } \\
\text { percentage }\end{array}$ \\
\hline Neat 3MOT & 7.5 & 49.1 \\
\hline Neat 3HOT & 3.3 & 52.6 \\
\hline Neat EDOT* & 3.0 & 27 \\
\hline Hexane-loaded 3MOT & 2.3 & 49.4 \\
\hline Hexane-loaded 3HOT & 0.8 & 47.5 \\
\hline Hexane-loaded EDOT & 6.7 & 40.7 \\
\hline $\begin{array}{c}\text { Hexane-loaded EDOT not } \\
\text { heat-treated }\end{array}$ & 0.4 & 32 \\
\hline
\end{tabular}

Table 1. Elemental mass percentage of copper and sulfur as measured by SEM/EDS in MOF samples.

EDS elemental composition analyses are not quantitative in a porous and nonhomogeneous material such as our composites. However, the presence of a strong sulfur signal is a good qualitative indication of a substantial amount of polythiophene present in the composite, whether in core-shell or filled form. In all cases, both neat and hexane-filled, the polymerized samples show a substantial sulfur signal in EDS. (Table 1) For the non-polymerized EDOT sample, only a faint sulfur signal is visible, likely because of evaporation of the unreacted monomer in the evacuated SEM chamber. On the other hand, the low sulfur signal of both 3HOT composites is consistent with the bulkier monomer diffusing less readily into the MOF pores, ${ }^{17}$ resulting in a lower overall degree of modification with P3HOT.

SEM imaging showed a change in the visible morphology of the MOF after sorption and heating of monomers. (Figure 3) A blend of octahedral and hexagonal crystals were present in the pristine material, but the composite after treatment was entirely in the hexagonal form. This phenomenon was reasonable in light of the PXRD pattern (Figure 4) which shows the increasing proportion of the (111) plane in the reacted material. This change is reasonable, since literature on HKUST-1 suggests that the the (111) face has a lower surface energy, resulting in a more stable structure. ${ }^{18}$ However, the mild heat applied to these samples is substantially lower than that required to effect a 
change in the morphology of HKUST-1, which is stable up to $260^{\circ} \mathrm{C}$. SEM of the intermediate material, (Figure 3, top) in which monomer was loaded but not yet heat-treated, suggests an explanation for this conversion. Visibly etched octahedral crystals can be seen in the micrographs of the intermediate material. The polymerization reaction releases both $\mathrm{H}^{+}$ions and electrons into the HKUST-1 lattice. Both species have the capacity to etch the lattice by protonation of linker ends and introduction of $\mathrm{Cu}(\mathrm{I})$ defects respectively. ${ }^{19,20}$ The partially degraded HKUST-1 is recrystallized by heating, resulting in full conversion to the more stable ${ }^{21}$ hexagonal form with a largerproportion of the (111) face. The barrier to annealing of HKUST-1 is likely reduced by the presence of defects introduced during the monomer/MOF reaction, which accounts for the structural change during heating. However, we cannot guarantee that defects alone are responsible for the ready conversion of the crystal form. The presence of guests within the MOF pores may also affect the barrier to recrystallization, since TGA analysis of MOF host-guest structures sometimes show a change at a lower temperature than the pristine MOF.

We also attempted to initiate polymerization of hexane-loaded alkoxythiophenes in HKUST-1 by external application of iodine vapor, similarly to a reported approach for terthiophene in other MOFs. However, this technique resulted in near-complete decomposition of this relatively fragile MOF, (Figure S5) probably due to the formation of HI byproduct since molecular iodine alone does not degrade HKUST-1. ${ }^{22,23}$ 

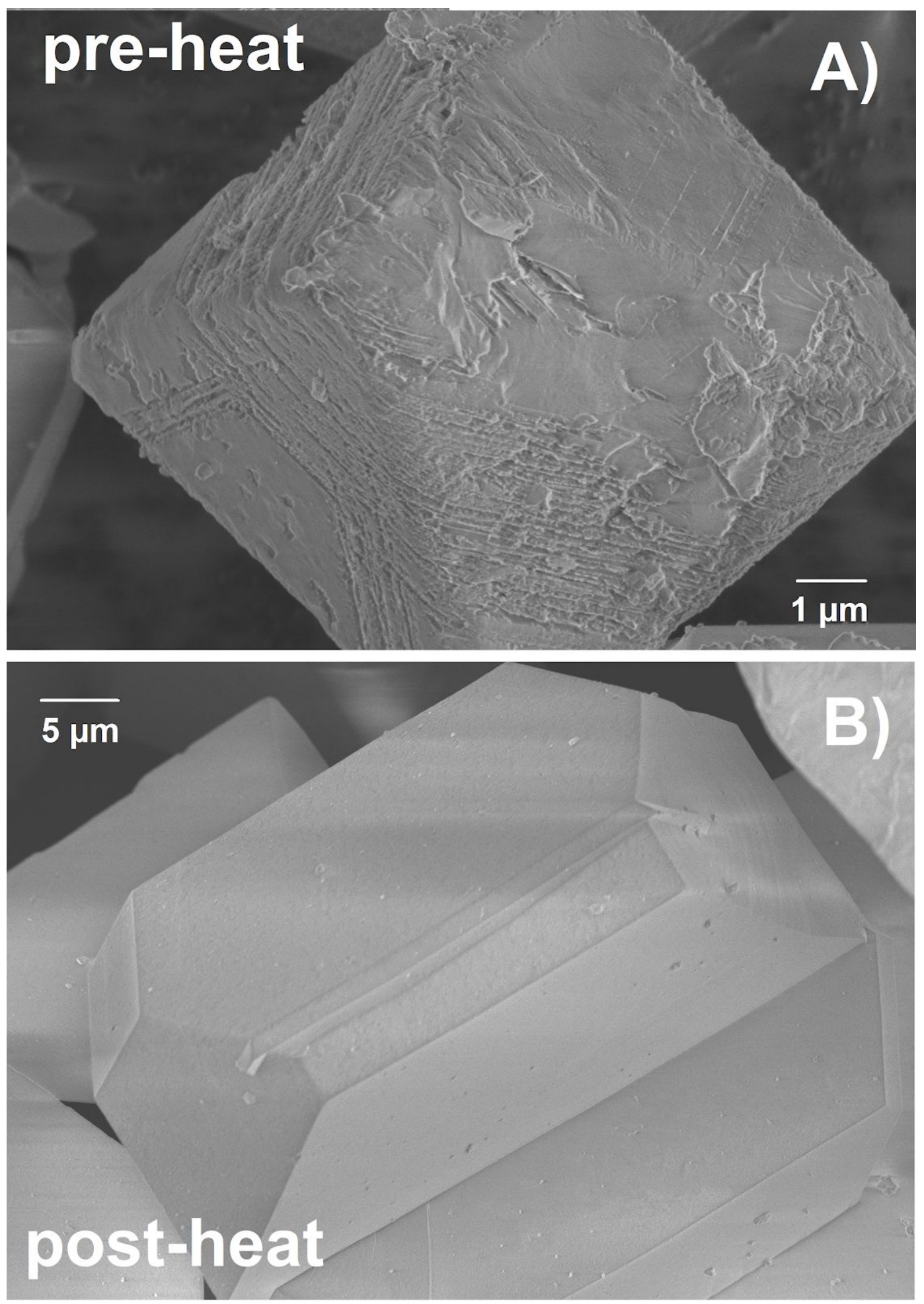

Figure 3. HKUST-1 in the presence of EDOT without heating leads to partial etching of the MOF, which upon heating results in a hexagonally faceted crystal form.

PXRD of pristine HKUST-1 and composites is consistent with retention of long-range MOF structure after composite formation, both in the neat and hexane-loaded composites. (Figure 4) In each composite for which significant reaction/modification (based on IR, BET, and EDS measurements) occurred, significant changes in the intensity are seen in the very low diffraction 
angle MOF peaks. The modified materials show a sharp increase in the ratio of the (002) peak to the (111) peak, based on literature peak assignments, ${ }^{19,24}$ consistent with our observation of the etching-and-recrystallization phenomenon seen in Figure 3. Smaller monomers EDOT and 3MOT result in a near-complete disappearance of the (111) peak, while this peak is still seen with significant intensity in the bulkier hexyloxy monomer 3HOT. In EDOT-based composite, the (002) peak is reduced in intensity as well, while in 3MOT composite it is still strong. Generally speaking, composites prepared with hexane-loaded monomers display this intensity change to a much greater degree than those prepared with neat monomer, consistent with our hypothesis that a primarily core-shell structure exists in the latter, e.g., that the interior of MOF crystals remains unmodified with the use of neat monomers. The etching of the surface of the HKUST-1 MOF while reacting with the framework is quite reasonable in light of ours and others' previous observation ${ }^{25,26}$ that reduction of the MOF by arenes can decompose the MOF. However, it surprised us that the etching disappeared in the heated, reacted material. Since PXRD reveals etching of the (002) face preferentially in the heated material, it seems that the defects induced by etching are healed by a recrystallization process at the surface.

The observation of this recrystallization process induced by exposure to alkoxythiophene monomers followed by heating is interesting and unprecedented. Since the MOF is heated during activation (before addition of monomer) to higher temperatures than that used to prepare the composite, the changes observed in crystal morphology are certainly enabled by the partial etching of the as-prepared MOF. We cannot conclude based on these limited observations whether the changes were catalyzed by the presence of $\mathrm{H}^{+}$ions, $\mathrm{Cu}(\mathrm{l})$ defects, the combination of the two, or the effects of filling HKUST-1 with a electron-rich organic guest polymer, but we will examine this question in future work. 

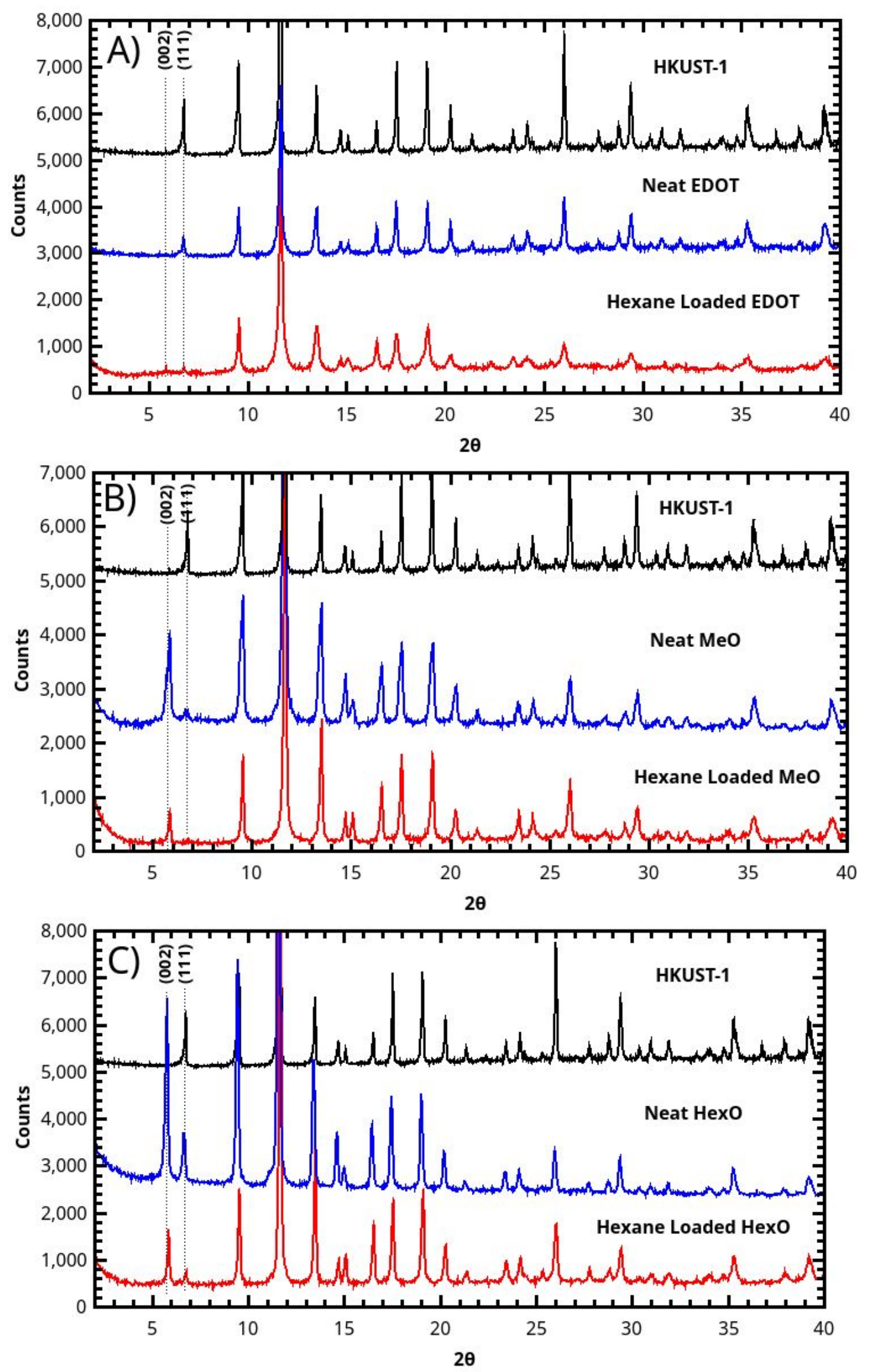

Figure 4. PXRD of HKUST-1 after reaction with neat and hexane-loaded alkoxythiophenes show that the crystal structure of the MOF is retained, but that the intensities of the (002) and (111) peaks (dashed lines) are altered by the reaction. 
Nitrogen-adsorption experiments fit using the BET isotherm showed that all alkoxythiophenes reacted with HKUST-1 to partially or completely fill its pores. (Table 2) In particular, EDOT and 3MOT composites, after reaction, had essentially the entire free volume of the MOF filled. 3HOT, while not as complete as the smaller monomers, still had roughly $5 / 6$ of the volume $(0.105 \mathrm{cc} / \mathrm{g}$ vs. $0.623 \mathrm{cc} / \mathrm{g}$ for pristine HKUST-1) filled after reaction. In contrast, direct combination of the liquid monomers with HKUST-1 gave a lower volume fraction filled for all three alkoxy monomers. SEM of the material from neat 3MOT shows a surface layer of polymer (Figure S3) while the neat 3HOT composite shows small surface features of retained polymer as well (Figure S4). It is likely that the neat 3HOT composite lost much of its surface polymer on washing with hexanes, since P3HOT would be much more soluble in organic solvents than the short-chain polymers.

\begin{tabular}{|c|c|c|}
\hline Composite & $\begin{array}{l}\text { Composite surface area } \\
\left(\mathrm{m}^{2} / \mathrm{g}\right)\end{array}$ & $\begin{array}{l}\text { Composite free volume } \\
\left(\mathrm{cm}^{3} / \mathrm{g}\right)\end{array}$ \\
\hline HKUST-1 & 2758 & 0.623 \\
\hline HKUST-1/neat EDOT* & 619 & 0.335 \\
\hline $\begin{array}{l}\text { HKUST-1/hexane-loaded } \\
\text { EDOT }\end{array}$ & 5.2 & 0.010 \\
\hline HKUST-1/neat MeO & 45.6 & 0.099 \\
\hline HKUST-1/hexane-loaded MeO & 11.5 & 0.021 \\
\hline HKUST-1/neat HexO & 684.4 & 0.273 \\
\hline $\begin{array}{l}\text { HKUST-1/hexane-loaded } \\
\text { HexO }\end{array}$ & 87.4 & 0.105 \\
\hline
\end{tabular}

* - From prior work. ${ }^{5}$ 


\section{Hexane-loaded EDOT}
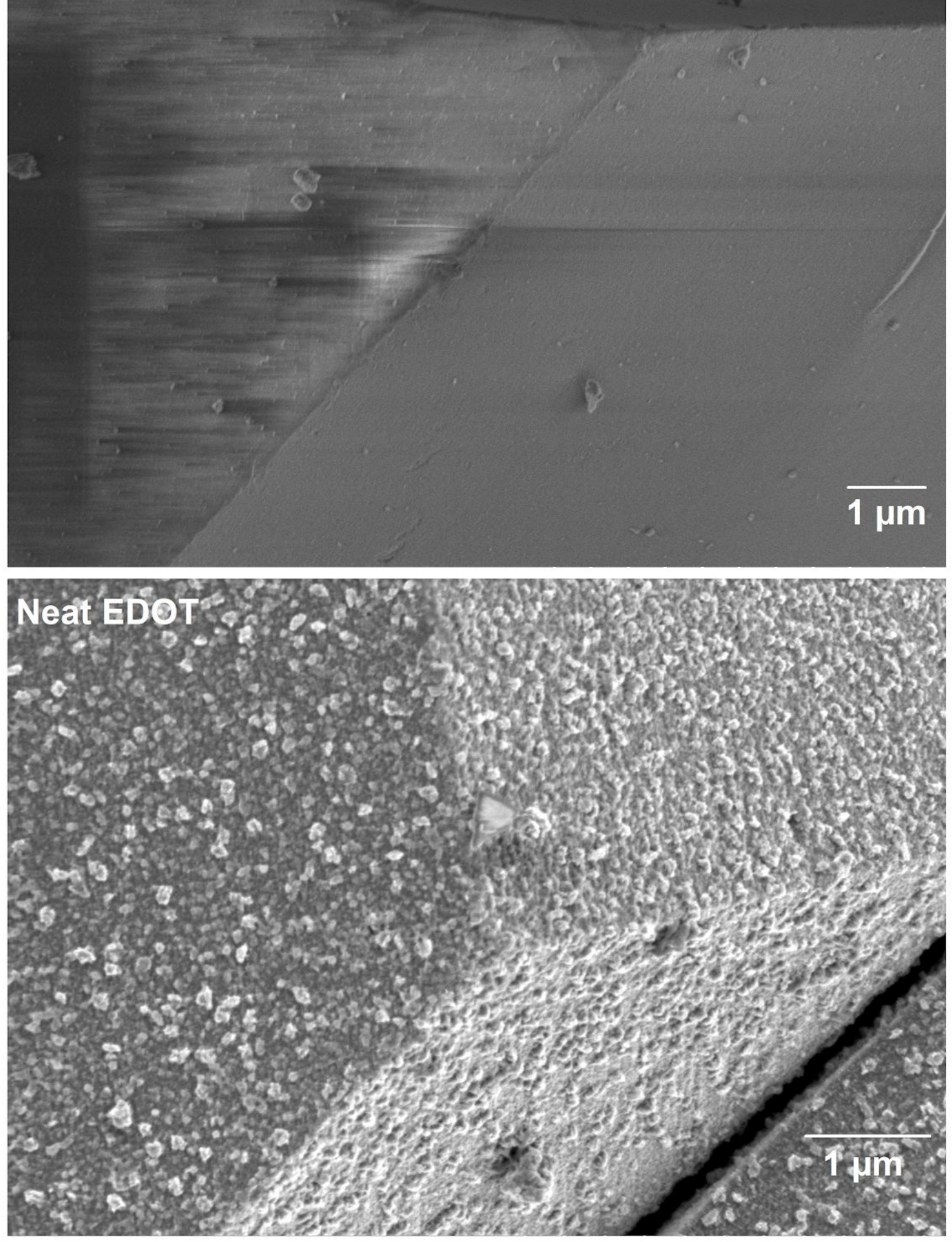

Figure 5. SEM of HKUST-1/PEDOT composites prepared using EDOT solution in hexanes (top) and neat EDOT (bottom). The neat composite shows substantial surface-bound polymer. 
We can directly observe the formation of the PEDOT 'shell' (and the absence of such a shell in the hexane-loaded composite) using SEM and AFM. (Figure 5 and S1-S2). A similar pattern holds for 3MOT, with the hexane-loaded composite being nearly completely filled by BET and showing high sulfur content by EDS (Table 1) without superficial polymer coating on SEM (Figure S6). On the other hand, a rough surface corresponding to surface polymer can be seen in the neat 3MOT composite. (Figure S3) This finding is consistent with ATR-IR spectroscopy of composites showing stronger conjugated polymer peaks for the neat composites than the hexane-loaded ones, despite similar or greater loading of polymer in the hexane-loaded composites, since the ATR technique preferentially detects surface matter. The overall picture painted by combined characterization of the two methods is that hexane-loaded HKUST-1 incorporates poly(alkoxy)thiophene inclusions internally, while HKUST-1 reacted directly with a neat polythiophene is coated on the surface.

\section{Conclusions}

Highly electron-rich alkoxythiophenes react at the surface of the redox-active MOF HKUST-1, self-limiting their ability to fill the pores of the MOF. By a two-step process of absorbing the MOF in hexanes, followed by heating to induce reaction, these highly reactive monomers can be coaxed to form filled composites with HKUST-1. In the larger 3-hexyloxythiophene, the bulk of the monomer hinders diffusion into the MOF pores. These results may aid materials researchers in the design of electronic metamaterials based on highly electron-rich conjugated polymers.

\section{Author Contributions}

W.J., C.M.S, and N.M. performed experiments and analyzed data. N.M. wrote the manuscript. The manuscript has been approved by all authors.

\section{References}

(1) Farha, O. K.; Hupp, J. T. Rational Design, Synthesis, Purification, and Activation of Metal-Organic Framework Materials. Acc. Chem. Res. 2010, 43 (8), 1166-1175. https://doi.org/10.1021/ar1000617.

(2) Liu, B.; Vellingiri, K.; Jo, S.-H.; Kumar, P.; Ok, Y. S.; Kim, K.-H. Recent Advances in Controlled Modification of the Size and Morphology of Metal-Organic Frameworks. Nano Res. 2018, 11 (9), 4441-4467. https://doi.org/10.1007/s12274-018-2039-3.

(3) Suh, M. P.; Park, H. J.; Prasad, T. K.; Lim, D.-W. Hydrogen Storage in Metal-Organic Frameworks. Chem. Rev. 2012, 112 (2), 782-835. https://doi.org/10.1021/cr200274s.

(4) Murray, L. J.; Dincă, M.; Long, J. R. Hydrogen Storage in Metal-Organic Frameworks. Chem. Soc. Rev. 2009, 38 (5), 1294-1314. https://doi.org/10.1039/B802256A.

(5) Marshall, N.; James, W.; Fulmer, J.; Crittenden, S.; Thompson, A. B.; Ward, P. A.; Rowe, G. T. Polythiophene Doping of the Cu-Based Metal-Organic Framework (MOF) HKUST-1 Using Innate MOF-Initiated Oxidative Polymerization. Inorg. Chem. 2019, 58 (9), 5561-5575. https://doi.org/10.1021/acs.inorgchem.8b03465.

(6) Uemura, T.; Kadowaki, Y.; Yanai, N.; Kitagawa, S. Template Synthesis of Porous Polypyrrole in 3D Coordination Nanochannels. Chem. Mater. 2009, 21 (18), 4096-4098. https://doi.org/10.1021/cm901361m.

(7) Haldar, R.; Sen, B.; Hurrle, S.; Kitao, T.; Sankhla, R.; Kühl, B.; Welle, A.; Heissler, S.; Brenner-Weiß, G.; Thissen, P.; Uemura, T.; Gliemann, H.; Barner-Kowollik, C.; Wöll, C. Oxidative Polymerization of Terthiophene and a Substituted Thiophene Monomer in Metal-Organic Framework Thin Films. Eur. Polym. J. 2018, 109, 162-168. https://doi.org/10.1016/j.eurpolymj.2018.09.040.

(8) Yanai, N.; Uemura, T.; Ohba, M.; Kadowaki, Y.; Maesato, M.; Takenaka, M.; Nishitsuji, S.; Hasegawa, H.; Kitagawa, S. Fabrication of Two-Dimensional Polymer Arrays: Template Synthesis of Polypyrrole between Redox-Active Coordination Nanoslits. Angew. Chem. Int. Ed. 2008, 47 (51), 9883-9886.

https://doi.org/10.1002/anie.200803846.

(9) McCann, G. F.; Millar, G. J.; Bowmaker, G. A.; Cooney, R. P. Formation of Polypyrrole and Polythiophene within 
Cu2+- and H+-Mordenite Hosts Studied by EPR and UV-VIS Spectroscopy. J. Chem. Soc. Faraday Trans. 1995, 91 (23), 4321-4328. https://doi.org/10.1039/FT9959104321.

(10) Jiao, Y.; Chen, G.; Chen, D.; Pei, J.; Hu, Y. Bimetal-Organic Framework Assisted Polymerization of Pyrrole Involving Air Oxidant to Prepare Composite Electrodes for Portable Energy Storage. J. Mater. Chem. A 2017, 5 (45), 23744-23752. https://doi.org/10.1039/C7TA07464F.

(11) Zhan, W.; Kuang, Q.; Zhou, J.; Kong, X.; Xie, Z.; Zheng, L. Semiconductor@Metal-Organic Framework Core-Shell Heterostructures: A Case of ZnO@ZIF-8 Nanorods with Selective Photoelectrochemical Response. J. Am. Chem. Soc. 2013, 135 (5), 1926-1933. https://doi.org/10.1021/ja311085e.

(12) Zhao, H.-X.; Zou, Q.; Sun, S.-K.; Yu, C.; Zhang, X.; Li, R.-J.; Fu, Y.-Y. Theranostic Metal-Organic Framework Core-Shell Composites for Magnetic Resonance Imaging and Drug Delivery. Chem. Sci. 2016, 7 (8), 5294-5301. https://doi.org/10.1039/C6SC01359G.

(13) Worrall, S. D.; Bissett, M. A.; Hirunpinyopas, W.; Attfield, M. P.; Dryfe, R. A. W. Facile Fabrication of Metal-Organic Framework HKUST-1-Based Rewritable Data Storage Devices. J. Mater. Chem. C 2016, 4 (37), 8687-8695. https://doi.org/10.1039/C6TC03496A.

(14) Xu, B.; Noh, S.; Thompson, B. C. Fine Tuning of Polymer Properties by Incorporating Strongly Electron-Donating 3-Hexyloxythiophene Units into Random and Semi-Random Copolymers. Macromolecules 2014, 47 (15), 5029-5039. https://doi.org/10.1021/ma5012107.

(15) Argun, A. A.; Aubert, P.-H.; Thompson, B. C.; Schwendeman, I.; Gaupp, C. L.; Hwang, J.; Pinto, N. J.; Tanner, D. B.; MacDiarmid, A. G.; Reynolds, J. R. Multicolored Electrochromism in Polymers: Structures and Devices. Chem. Mater. 2004, 16, 4401-4412. https://doi.org/10.1021/cm049669I.

(16) Moraes, B. R.; Campos, N. S.; Izumi, C. M. S. Surface-Enhanced Raman Scattering of EDOT and PEDOT on Silver and Gold Nanoparticles. Vib. Spectrosc. 2018, 96, 137-142. https://doi.org/10.1016/j.vibspec.2018.04.006.

(17) Heinke, L. Diffusion and Photoswitching in Nanoporous Thin Films of Metal-Organic Frameworks. J. Phys. Appl. Phys. 2017, 50 (19), 193004. https://doi.org/10.1088/1361-6463/aa65f8.

(18) Liu, Q.; Yang, J.-M.; Jin, L.-N.; Sun, W.-Y. Metal Ion Induced Porous HKUST-1 Nano/Microcrystals with Controllable Morphology and Size. CrystEngComm 2016, 18 (22), 4127-4132.

https://doi.org/10.1039/C5CE02497H.

(19) Doan, H.; Sartbaeva, A.; Eloi, J.-C.; Davis, S.; Ting, V. Defective Hierarchical Porous Copper-Based Metal-Organic Frameworks Synthesised via Facile Acid Etching Strategy. Sci. Rep. 2019, 9, 10887. https://doi.org/10.1038/s41598-019-47314-1.

(20) Jia, K.; Ye, J.; Zhuang, G.; Zhuang, Z.; Yu, Y. Well-Defined Cu2O/Cu3(BTC)2 Sponge Architecture as Efficient Phenolics Scavenger: Synchronous Etching and Reduction of MOFs in Confined-pH NH3 H2O. Small 2019, 15. https://doi.org/10.1002/smll.201805478.

(21) Jiang, H.; Lu, B.; Ma, L.; Yuan, X. Effect of Crystal Form Control on Improving Performance of Cu3(BTC)2 Immobilized Phosphotungstic Acid in Esterification of Cyclohexene with Formic Acid. Catal. Lett. 2020, 150 (6), 1786-1797. https://doi.org/10.1007/s10562-019-03090-1.

(22) Zhao, Q.; Zhu, L.; Lin, G.; Chen, G.; Liu, B.; Zhang, L.; Duan, T.; Lei, J. Controllable Synthesis of Porous Cu-BTC@polymer Composite Beads for lodine Capture. ACS Appl. Mater. Interfaces 2019, 11 (45), 42635-42645. https://doi.org/10.1021/acsami.9b15421.

(23) Valizadeh, B.; Nguyen, T. N.; Smit, B.; Stylianou, K. C. Porous Metal-Organic Framework@Polymer Beads for lodine Capture and Recovery Using a Gas-Sparged Column. Adv. Funct. Mater. 2018, 28 (30), 1801596. https://doi.org/10.1002/adfm.201801596.

(24) Langseth, E.; Swang, O.; Arstad, B.; Lind, A.; Cavka, J. H.; Jensen, T. L.; Kristensen, T. E.; Moxnes, J.; Unneberg, E.; Heyn, R. H. Synthesis and Characterization of Al@MOF Materials. Mater. Chem. Phys. 2019, 226, 220-225. https://doi.org/10.1016/j.matchemphys.2019.01.009.

(25) El-Hankari, S.; Huo, J.; Ahmed, A.; Zhang, H.; Bradshaw, D. Surface Etching of HKUST-1 Promoted via Supramolecular Interactions for Chromatography. J. Mater. Chem. A 2014, 2 (33), 13479-13485. https://doi.org/10.1039/C4TA02568G.

(26) Zhou, C.; Li, A.; Wang, D.; Pan, E.; Chen, X.; Jia, M.; Song, H. MOF-Templated Self-Polymerization of p-Phenylenediamine to a Polymer with a Hollow Box-Assembled Spherical Structure. Chem. Commun. 2019, 55 (28), 4071-4074. https://doi.org/10.1039/C9CC01081E. 